\title{
La dignidad humana y el derecho de disposición sobre el propio cuerpo. Reflexiones a partir del rechazo de tratamientos médicos y los acuerdos de maternidad subrogada
}

\begin{abstract}
Human Dignity and the Right to Selfdetermination over One's Own Body. Considerations Regarding the Refusal of Medical Treatment and Surrogacy

Agreements
\end{abstract}

\begin{abstract}
A dignidade humana e o direito de disposição no próprio corpo. Reflexões a partir da recusa a tratamentos médicos e acordos de maternidade por sub-rogação
\end{abstract}

Mercedes Ales Uría*

\footnotetext{
* $\quad$ https://orcid.org/0000-0003-2800-7696. Universidad del Salvador, Centro de Estudios Macroeconómicos de Argentina (CEMA), Universidad de Buenos Aires, Argentina. mercedesales@derecho.uba.ar
}

Recibido: 06/03/2017. Envío a pares: 06/03/2017 Aprobado por pares: 23/05/2017. Aceptado: 24/09/2019

DOI: 10.5294/dika.2020.29.1.2 


\section{Resumen}

En este artículo se abordan las implicaciones prácticas de la noción de dignidad humana como elemento intrínseco del concepto de persona y fundante de los derechos humanos. Estas implicaciones se proyectan sobre las decisiones referidas al rechazo de terapias y a los acuerdos de alquiler de vientres para valorar su ordenación o contradicción con la noción de dignidad humana ensayada. En primer lugar, se ensaya una definición con base en instrumentos legales internacionales, europeos y argentinos. Se hace una revisión histórica de la fundamentación de los derechos y la dignidad del ser humano en la filosofía clásica. A partir de esas conclusiones, se abordan los casos de negativa a tratamientos médicos o disposición sobre el propio cuerpo en virtud de una objeción de conciencia, y los supuestos de madres subrogantes tanto en casos altruistas como de alquiler comercial de vientres. En el primer caso, se analiza la coherencia con un sentido de dignidad fundante; en el segundo, se entiende contrario a la afirmación de la dignidad humana. La gestación por sustitución se concibe como un estereotipo de género que reduce a la mujer y al niño a objetos de derechos de terceros.

\section{Palabras clave}

Dignidad; derechos del niño; disposición; disposición sobre el propio cuerpo; objeción de conciencia; tratamiento médico; alquiler de vientres; gestación subrogada: género; vulnerabilidad. 


\section{Abstract}

This paper seeks to elaborate on the practical implications of an understanding of human dignity both as an element intrinsic to the concept of person and as a foundation of human rights. These implications are projected on decisions pertaining to the refusal of medical treatment and surrogacy agreements in order to assess their alignment with or contradiction of the proposed notion of human dignity. Firstly, a definition is provided on the basis of international legal instruments on human rights as well as European and Argentine bills of rights. This is followed by a historical review of a classic philosophy approach to the fundamentals of human rights and human dignity. From the conclusions drawn, cases of refusal of medical treatment and self-determination over one's own body under conscientious objection and both altruistic and commercial surrogacy agreements are addressed. The former seems coherent with a founding sense of dignity; the latter is understood as contrary to the affirmation of dignity. Surrogate maternity practices are envisioned as a gender stereotype that reduces the woman and child to objects of third parties' rights.

\section{Keywords}

Dignity; rights of the child; self-determination; self-determination over one's own body; conscientious objection; medical treatment; surrogacy; surrogate pregnancy; gender; vulnerability. 


\section{Resumo}

Neste artigo, são abordadas as implicações práticas da noção de dignidade humana como elemento intrínseco do conceito de pessoa e fundante dos Direitos Humanos. Essas implicações são projetadas sobre as decisões referidas à recusa a terapias e a acordos de maternidade por sub-rogação ("barriga de aluguel") para avaliar sua ordenação ou contradição com a noção de dignidade humana ensaiada. Em primeiro lugar, é ensaiada uma definição com base em instrumentos legais internacionais, europeus e argentinos. Faz-se uma revisão histórica da fundamentação dos direitos e da dignidade do ser humano na Filosofia clássica. A partir destas conclusões, são abordados os casos de negação a tratamentos médicos ou disposição sobre o próprio corpo devido a uma objeção de consciência e às suposições de mães sub-rogadas tanto em casos altruístas quanto em de aluguel comercial de útero. No primeiro caso, é analisada a coerência com um sentido de dignidade fundante; no segundo, entende-se contrário à afirmação da dignidade humana. A gestação por substituição é concebida como um estereótipo de gênero que reduz a mulher e a criança a objetos de direitos de terceiros.

\section{Palavras-chave}

Dignidade; direitos da criança; disposição; disposição no próprio corpo; objeção de consciência; tratamento médico; "barriga de aluguel"; sub-rogação gestacional; gênero; vulnerabilidade. 
Sumario: Introducción. 1. La dignidad humana en un sentido completo e inherente. 1.1. Un breve recorrido histórico-filosófico por la noción de dignidad. 1.2. La dignidad humana como fundamento de los derechos. 1.3. Libre desarrollo de la personalidad: el cauce de la dignidad humana. 2. La Dignidad y la disposición sobre el propio cuerpo. 2.1. El rechazo de tratamientos médicos. Objeción de conciencia o eutanasia. 2.2. Maternidad subrogada. Jurisprudencia comparada. 3. Conclusiones.

\section{Introducción}

El debate contemporáneo en materia de derechos humanos y filosofía del derecho ha visto un renovado interés en el estudio de la dignidad humana, sus implicaciones, exigencias y rol en la fundamentación y el desarrollo de los derechos. El presente trabajo pretende reflexionar en torno a un caso concreto de aplicación de este concepto en situaciones en las que se ha analizado el alcance de la potestad jurídica de disposición sobre el propio cuerpo a la luz de la autonomía y el desarrollo de la personalidad. Para ello, se han tomado ejemplos en materia de rechazo de terapéuticas médicas en el contexto de riesgo para la vida del paciente, y precedentes recientes en materia de maternidad subrogada.

Para abordar el análisis de las situaciones descritas, se explicita primero el entendimiento de la dignidad humana, su alcance y lugar en la elaboración de los derechos fundamentales, en los que se fundamenta el presente trabajo. A partir de estos presupuestos es que se aborda la temática con la finalidad de contribuir en la dilucidación de un concepto fuerte que realce la centralidad de la persona en el ordenamiento jurídico nacional e internacional. En este sentido, el trabajo aplicará una metodología descriptiva y de lógica deductiva. Se procederá a la elaboración de una definición de "dignidad humana" para postular el carácter fundante de los derechos humanos. Seguidamente, se elaborarán tres criterios de consideración de la aplicación del estándar de dignidad al accionar humano. A la luz de la definición alcanzada y los criterios desarrollados, se evaluará la negativa a ciertos tratamientos médicos sobre la base de creencias personales y el uso del propio cuerpo para un acuerdo de gestación subrogada. Finalmente, se postularán las conclusiones del desarrollo.

\section{La dignidad humana en un sentido completo e inherente}

La reflexión en torno a la dignidad como fundamento de los derechos y, más concretamente, en su articulación como fundamento y límite para el libre desarrollo de la personalidad a través de la disposición sobre el propio cuerpo comienza con la Declaración Universal de Derechos del Hombre (DUDH). Este instrumento configura a la dignidad como piedra angular de su sistema $\mathrm{y}$ fundamento de todos los derechos. Lo mismo hace el Pacto Internacional de Derechos Civiles y Políticos (PIDCP) que afirma, en su Preámbulo, que los derechos tutelados se derivan de la dignidad inherente a la persona humana. 
La Unión Europea ha incluido a la dignidad en el articulado de la Carta de Derechos Fundamentales en su artículo II-61. Se trata, entonces, de uno de los valores sobre los cuales se asienta la UE, de acuerdo con el artículo 2 del Tratado de Lisboa.

También se podría citar el ejemplo de la Constitución Española de 1978, que consagró como fundamento del orden público y la paz social el libre desarrollo de la personalidad y la dignidad de la persona. ${ }^{1}$ Asimismo, es frecuente citar en este tópico el artículo 1.1 de la Ley Fundamental de Bonn ${ }^{2}$ en tanto, en la teoría tradicionalmente sostenida por diversos autores alemanes, esta norma contiene un "derecho a la dignidad" que se traduce en la existencia conjunta de un "derecho general de la personalidad" junto con aquellos de la personalidad particulares o especiales. ${ }^{3}$ Este primer derecho general es el fundamento de todos los derechos subjetivos y su objeto es una parte destacada de la esfera de la personalidad. ${ }^{4}$

Los valores de libertad e igualdad sobre los que se asienta la primacía de los derechos fundamentales en un Estado de derecho aparecen vinculados a la consideración del hombre como persona. ${ }^{5}$ Esto se traduce en la supremacía del valor de la dignidad humana como elemento central en los tratados de derechos humanos y en la constitución de gran número de Estados. Este reconocimiento de la dignidad esencial o inherente de todo ser humano implica su derecho a ser tratado de forma igual y sin discriminaciones. ${ }^{6}$

No obstante, la dificultad radica, como ocurre con los estándares jurídicos abiertos, en definir qué se entiende por dignidad. Ningún texto legal lo define y queda, entonces, en el prudente entender y la intuición del intérprete develar cuáles son sus exigencias en cada caso concreto. Haciendo nuestra la elaboración del Tribunal Constitucional Español, se podría conceptualizar a la dignidad como

... un valor espiritual y moral inherente a todas las personas, un mínimo que debe preservarse de las vulneraciones por parte de los poderes públicos y los particu-

1 Véase en este punto a Pablo Lucas Verdú, “Comentario al art. 10”, en AA.VV., Constitución Española, Madrid, Centro de Estudios Constitucionales, 1979, p. 39.

2 En este particular se seguirá la clásica exposición de Robert Spaemann, "Sobre el concepto de dignidad humana", Revista Persona y Derecho 19 (1988), pp. 16 y ss.

3 Cfr. Carlos Rogel Vide, Bienes de la personalidad, derechos fundamentales y libertades públicas, Bolonia, Publicaciones del Real Colegio de España, 1985, pp. 33-35.

4 En Francisco De Castro y Bravo, "Los llamados derechos de la personalidad", Anuario de Derecho Civil (1959), pp. 1237, 1252. Esta teoría recobró fuerza después de la Segunda Guerra Mundial con la entrada en vigor de la Constitución de Bonn. Karl Larenz explica que, tras la guerra, se consideró insuficiente la protección de la personalidad humana otorgada hasta entonces, especialmente frente a los horrores cometidos durante dicha época. Después de los atropellos del régimen nacionalsocialista, la sociedad alemana se había sensibilizado frente a toda clase de menosprecio de la dignidad humana y la personalidad (Francisco De Castro Y Bravo, Derecho civil. Parte general, traducción y notas de Miguel IzQuierdo y Macías-Picavea, Madrid, 1978, pp. 155-160).

5 Véase Susana Salvador Gutiérrez, “Derecho a la identidad”, Actualidad Civil IV (1999), pp. 1469-1498.

6 Ibid., pp. 1470-71. 
lares. Se manifiesta en la autodeterminación consciente y responsable de la propia vida, en la autonomía individual y constituye el punto de partida para la existencia y especificidad de los demás derechos fundamentales.?

En una metavisión de los particulares instrumentos positivos que puedan servir de ejemplos, el concepto de dignidad que se acoge en las normas fundantes hace alusión a lo que en términos metafísicos se denomina dignidad completa e inherente (full inherent dignity) ${ }^{8}$ Ello en tanto se trata de una cualidad o valor de carácter permanente, incondicionado, indivisible e inviolable y que puede ser predicada de todo ser humano. Se entiende, entonces, que inherente es intercambiable con la noción de intrínseco y, por ende, hace alusión a lo que no puede ser separado de la sustancia en la que se da. En este caso, la dignidad es una cualidad que no hace al accidente de la persona, sino a su sustancia, en términos del pensamiento de la razón práctica. ${ }^{9}$

Fundamentalmente, la calidad de inherente o intrínseco del concepto de dignidad tiene dos grandes vertientes de exigencias deónticas: la primera, que no debe ser violentada, y la segunda, que no puede ser dañada y que es imposible disociarla de la noción de persona. Ambas exigencias se proyectan hacia la sociedad, en la forma del Estado y los poderes públicos, e internamente hacia el portador que no puede desprenderse de su calidad de digno. ${ }^{10}$

Es una referencia que enmarca al sistema legal, y que exige que el Estado respete y promocione todo aquello que la haga posible, a la vez que remueva todos los obstáculos para su pleno desarrollo. El constituyente de más de 150 países del mundo consideró suficiente la referencia a la dignidad humana para basar en ella derechos que quiere reconocer, a fin de garantizar los atributos de la personalidad. ${ }^{11}$

Como última aclaración en este apartado, vale destacar que el carácter intrínseco de la dignidad en relación con lo humano es considerado con independencia de los supuestos en los que ciertos miembros de la especie humana carezcan en el acto de la plenitud de los elementos que se utilizan para describir a la dignidad, principalmente, en razón de su edad o salud. No obstante,

$7 \quad$ En sentencias de 24 de mayo de 1982, 11 de abril de 1985 y 28 de febrero de 1994, entre otras.

8 Véase Lucy Micheal “Defining dignity and its place in human rights", The New Bioethics 20 (1) (2014), pp. 12-34, p. 13.

9 Véase Renato Rabbi-Baldi Cabanillas, Teoría del Derecho, $4^{a}$ ed., Buenos Aires, Ábaco de Rodolfo de Palma, 2015, pp. 56-59. En un brevísimo racconto histórico, cabría mencionar que la noción de "dignidad" comienza a vislumbrarse en sus raíces religiosas con el judaísmo y, posteriormente, el cristianismo a partir de la noción de imago Dei. Luego del desarrollo de los teólogos y filósofos medievales, en el renacimiento es Picco della Mirandola quien asocia este concepto con el libre albedrío. Kant, en los albores de la Modernidad, construye la noción de dignidad sobre la base de la racionalidad y la moralidad del hombre y, en consecuencia, el valor inconmensurable de todos los seres humanos. En este recorrido, sería injusto olvidar a Francisco de Vittoria y su labor en pos del igual reconocimiento de derechos, fundados en la dignidad, de los nativos americanos.

10 Ángeles Lara Aguado, El nombre en derecho internacional privado, Granada, Comares, 1998, pp. 76-77.

11 José Manuel Martín Bernal, "Los derechos de la personalidad en la Constitución Española”, Revista General de Legislación y Jurisprudencia s.d. (1979), p. 13. Véase Yechiel Michael BarILÁn, Human Dignity, Human Rights and Responsibility, Cambridge, Massachussets Institute of Technology Press, 2012, p. 2. 
se asume la visión de que aquellos que se ven disminuidos en estos aspectos -la autodeterminación consciente y responsable de la propia vida-son sujetos de especial protección en virtud de su vulnerabilidad que en nada menoscaba su dignidad. ${ }^{12}$

\subsection{Un breve recorrido histórico filosófico por la noción de dignidad}

Como se indicó en el apartado precedente, el concepto de "dignidad" es abierto. Ha sido objeto de discusión y estudio desde la Antigüedad clásica. Es, en simultáneo, uno de los grandes tópicos del pensamiento actual y también uno de sus grandes enigmas. Si bien, a los fines del trabajo que se presenta, se ha optado por una definición extraída de la jurisprudencia del máximo tribunal Constitucional Español,13 se hace a continuación una somera relación de antecedentes históricos en el devenir de las ideas de Occidente.

El primer antecedente del que se tiene noción es del sánscrito, el vocablo dec, que vendría a significar "ser conveniente" o "adecuado" a algo. Este término fue latinizado con el sufijo mus para formar el término decmus que derivó en dignus. ${ }^{14}$ De allí el castellano "dignidad" que, conforme la Real Academia Española, nos refiere a la cualidad de "digno", que es "merecedor de algo".

A partir de la Antigüedad podemos dividir el significado histórico del término en dos grandes temáticas: la consideración social y una cualidad intrínseca al ser. ${ }^{15}$ La primera es propia de Roma, en la que la dignidad se refería al posicionamiento social y a la función pública del sujeto. Así, se habla de la dignidad de determinados funcionarios; noción que luego es adoptada en el tratamiento de la realeza y de ciertos cargos en la misma Iglesia católica (así la dignidad máxima del Sumo Pontífice y de los cardenales y obispos). La segunda es propia del judeo-cristianismo a partir del helenismo, que consiste en una forma de ver al hombre como el centro del universo. Se lo ve como una criatura dotada de una particular esencia, fruto de haber sido creada por Dios a su imagen y semejanza, ser imago $\mathrm{Dei}^{16} \mathrm{~A}$ partir del renacimiento de la filosofía humanista, con autores como Picco della Mirandola y Giordano Bruno, el concepto de la dignidad como una cualidad propia del hombre e intrínseca a su naturaleza pasa a ser un tópico recurrente. Aunque, cabe señalar, la

12 Rabbi-Baldi, Teoría del Derecho, op. cit., con cita de Immanuel Kant, Fundamentación de la metafisica de las costumbres, Madrid, Espasa Calpe, trad. de M. García Morente, 1983, pp. 80 a 94. Se toma el concepto de ser humano digno con entera prescindencia de sus accidentes y resaltando el valor intrínseco e irreducible de toda persona. En idéntico sentido, Micheal, "Defining dignity and its place in human rights", op. cit., p. 16.

13 Véase nota al pie 7.

14 Véase el desarrollo de Gregorio Peces Barba, "La dignidad de la persona desde la filosofía del Derecho", en Cuadernos Bartolomé de las Casas 26 (2002), pp. 21-61.

15 Íñigo de Miguel Beriain, “Consideraciones sobre el concepto de dignidad humana”, Anuario de filosofia del derecho 21 (2004), pp. 189-192.

16 Rabbi-BALDi, Teoría del Derecho, op. cit., p. 62. 
noción de dignidad conserva una fuerte impronta religiosa ya que, en el abanico de autores, se la entiende como un don recibido del Creador. ${ }^{17}$

El concepto de dignidad se seculariza, por así decirlo, con la perenne contribución de Immanuel Kant que enriquece la historia de la filosofía occidental con la noción de lo digno como aquello que no tiene precio y la noción de que la cualidad de humano es en sí misma una dignidad. ${ }^{18}$ A partir de entonces, adentrándonos hacia la modernidad y posmodernidad, encontramos cada vez más nítidas dos corrientes de pensamiento. La una que estima que la dignidad hace referencia a "el valor intrínseco de la persona, derivado de una serie de rasgos que la hacen única e irrepetible, que es el centro del mundo y está centrada en el mundo". ${ }^{19} \mathrm{Y}$ la otra que entiende a esta cualidad en un sentido relacional, es decir que varía en función de los acontecimientos y es diferente para cada ser humano. ${ }^{20}$

\subsection{La dignidad humana como fundamento de los derechos}

Proclamada abstractamente, la dignidad no dice nada en términos legales, pero se refiere a todos los derechos, lo que llama a reflexionar en cuanto a su función en el plexo normativo. ${ }^{21}$ En palabras de Spaemann: "este concepto [la dignidad] no indica de modo inmediato un derecho humano específico, sino que contiene la fundamentación de lo que puede ser considerado como derecho humano en general". 22

Es a partir de la misma vaguedad con que se sitúa el concepto de dignidad, tanto en los instrumentos internacionales como en las cartas fundacionales paradigmáticas, que se ha postulado que poseería un doble carácter como el fundamento de los derechos y como derecho en sí. Por un lado, se presenta como valor de los sistemas normativos que la acogen y como principio de acción del Estado de derecho, vinculando a todos los poderes y organismos públicos. Por otro, la dignidad humana también se postula como derecho fundamental autónomo, aunque sin especificar en qué consiste este derecho.

17 Peces Barba, “La dignidad de la persona desde la filosofía del Derecho", op. cit., p. 30.

18 Se sigue el núcleo de lo expuesto por Immanuel KANT, Fundamentación para una metafisica de las costumbres, Madrid, Alianza Editorial, 2002. No obstante, se hace la aclaración de que muchos autores sostienen que la noción de dignidad humana en un sentido fundante precisa, necesariamente, de la nota de sacralidad. Es decir que, en última instancia, se trata de una idea metafísica-religiosa. Veáse, en particular, Horkheimer y Adorno.

19 Peces Barba, “La dignidad de la persona desde la filosofía del Derecho", op. cit., p. 65.

20 Este es diferente al planteamiento que hacen, entre otros, Robert Spaemann, en cuanto a la diferenciación de la naturaleza del hombre y la valoración de sus actos. Es decir, que puede sostenerse coherentemente que el hombre es un ser dotado de dignidad, pero capaz de realizar actos indignos a partir de su voluntad libre.

21 Fernando Garrido Falla, Comentarios a la Constitución, Madrid, Civitas, 1985, p. 187. En esta labor han de servir de guía la jurisprudencia de los tribunales, especialmente el TCE, y la atención al contexto sociocultural puesto que la valoración que la misma sociedad haga de un derecho y la interiorización de su contenido es una de las vías más directas para comprender su esencia.

Spaemann, "Sobre el concepto de dignidad humana", op. cit., p. 15. 
En relación con la segunda postulación, no puede obviarse que en la práctica legislativa y de los tribunales, lo que entendemos por dignidad humana se concreta en el contenido de cada uno de los derechos fundamentales que en ella encuentran su justificación. ${ }^{23}$ Los derechos inviolables les son reconocidos al hombre y a la mujer en virtud de su dignidad inherente y en pro del libre desarrollo de su personalidad. ${ }^{24}$ Esto tiene una gran virtualidad práctica en tanto, al ser la dignidad un principio absoluto e inderogable, es un coralario lógico que no cabe atentar contra ella con el pretexto de defender otros derechos. ${ }^{25}$ Desde esta óptica, no cabría realizar una ponderación o balance de la dignidad en hipotético conflicto con algún derecho fundamental. Al ser su fuente $u$ origen, se encuentra ontológica y lógicamente en otro plano de análisis. ${ }^{26}$

En este sentido, se afirma que la dignidad ocupa un rango fundamental y posee eficacia legitimadora, iluminadora y propulsora para afianzar instituciones, esclarecer ambigüedades, cubrir lagunas e integrar nuevas potencialidades. ${ }^{27}$ En otras palabras, la dignidad hace las veces de título de dominio de derechos que son inherentes a la condición de persona y que, de acuerdo con el momento histórico, el legislador o tribunal, se han denominado "humanos", "fundamentales" o "esenciales".28

En resumidas cuentas, la dignidad humana actúa como fuente de validez y legitimidad del ordenamiento jurídico en los aspectos que conciernen a los derechos fundamentales. ${ }^{29}$ Reconocer su carácter inherente y fundante nos recuerda que dichos derechos no existen en virtud de su reconocimiento por el Estado o cualquier autoridad externa. ${ }^{30}$

Los derechos fundamentales son el núcleo esencial e inviolable de derechos derivados de la misma naturaleza humana, que nadie ni nada debe cohibir, y que el Estado debe ayudar a preservar, ofreciendo las condiciones necesarias

23 Araceli Mangas Martín (dir.), Carta de los Derechos Fundamentales de la UE, Bilbao, Ed. Fundación BBVA, 2008, p. 128.

24 Ver sentencias del TCE de 11 de abril de 1985 y 27 de junio de 1990.

25 Mangas Martín, Carta de los Derechos Fundamentales de la UE, op. cit., p. 108.

26 Al referirme a la dignidad en sentido "ontológico" hago referencia al valor que le corresponde a la persona por el solo hecho de ser tal, con independencia de sus cualidades accidentales (también en sentido ontológico) y de su coyuntura vital. En qué consiste esta dignidad ontológica es, por supuesto, otro gran campo de debate, pero, esencialmente, se sigue en este trabajo a Kant al entender que los rasgos característicos de la dignidad humana consisten en la capacidad del ser humano de emitir juicios morales, su libertad para decidir acerca de sus acciones externas e internas y la capacidad intelectual de intelegir la realidad sensible y no sensible.

27 Ibid., p. 100.

28 Rabbi-BALDi, Teoría del Derecho, op. cit., p. 163.

29 Klaus Dicke, "The founding function of human dignity in the Universal Declaration of Human Rights", en Kretzmer \& Klein, The Concept of Dignity in Human Rights Discourse, 111 at 118, en Jeremy Waldron, "Is dignity the foundation of human rights", New York University Public Law and Legal Theory Working Papers, Paper 374 (2013), en http://lsr.nellco.org/nyu_plltwp/374/?utm_source=lsr.nellco.org\%2Fnyu_ plltwp\%2F374\&utm_medium=PDF\&utm_campaign=PDFCoverPages

30 Véase por muchos Oscar SсHAснтеR, "Human dignity as a normative concept", American Journal of International Law 77 (1983), p. 848. 
para su realización. ${ }^{31}$ Deben ser respetados por los poderes políticos y también por las actividades individuales de los sujetos humanos. Son "aquellos derechos de la persona humana -considerada tanto en su aspecto individual como comunitario- que corresponden a esta por razón de su propia naturaleza [...] y que deben ser reconocidos y respetados por todo Poder o autoridad y toda norma jurídica positiva". ${ }^{32}$

Más allá de las notas y los caracteres propios de la dignidad humana recién listadas, y que podrían ser consideradas como clásicas en la tópica, cabe resaltar que la noción de fundante implica también un baremo de juicio para derivar derechos fundamentales a partir de ella. En otras palabras, al ser la dignidad humana el título fuente de los derechos fundamentales, todo derecho que se pretenda derivar de ella tendrá como criterios delimitadores la propia dignidad. ${ }^{33}$ La dignidad de la persona y sus derechos inviolables no son realidades estáticas en el mundo del derecho, sino que evolucionan de acuerdo con las exigencias sociales y los nuevos desafíos en un proceso histórico de gradual despliegue de potencialidades.

El concepto de dignidad como fundamento de los derechos hace las veces de fuente de inspiración para el legislador y, como tal, debe ser entendida como una pauta orientadora para los poderes públicos. Se trata de una pauta programática que sirve de punto de enlace entre los valores ético-sociales y las normas positivas que conforman el Estado. Pero, a su vez, posee peso vinculante y exigencia de ejecución en cada uno de los derechos que de ella fluyen, aunque precisa de desarrollo

31 José Castán Tobeñas, Los derechos del hombre, 2 a ed., Madrid, Reus, 1976, pp. 12-15. El Estado de derecho, es decir, el Estado sometido al ordenamiento jurídico, implica un elenco de derechos sobre los cuales existe un consenso generalizado puesto que se estiman necesarios para la existencia de una democracia. Es usual hablar de generaciones de derechos de acuerdo con la época en que cada grupo fue reivindicado y de cuyas ideas se nutrió. Así, tenemos una primera generación que se correspondía con el Estado liberal de derecho y que consistió en limitar jurídicamente al poder para garantizar la libertad individual. Esta primera generación está representada por los derechos individuales más básicos: vida, libertad, propiedad, seguridad, participación política limitada. Es una libertad que resiste los embates del poder, pero que no exige un desarrollo y promoción de aspiraciones. La segunda generación se corresponde con la del Estado liberal cercado e incorpora el sufragio - aunque solo para los hombres- y ciertas libertades grupales como el derecho de asociación. La tercera generación es la que nace al amparo del Estado social de derecho o Wellfare State, en el que se da una fuerte interacción entre el Estado y los particulares. Supone la integración de los derechos económicos y sociales y los derechos de prestación, es decir, la acción positiva del Estado para lograr una igualdad real por sobre la formal. El Estado está obligado a crear y mantener las condiciones materiales para el desenvolvimiento de determinados derechos como la educación, la tutela judicial, la vivienda, etc.

Finalmente, en la doctrina se menciona la cuarta generación de derechos que han tenido acogida tanto en los textos fundamentales de las naciones como en los instrumentos internacionales. No responde a un quiebre sustancial en el modelo de Estado social de derecho, sino a una evolución de este debido, mayormente, a las reivindicaciones de grupos de ciudadanos y a las transformaciones científicas y técnicas aplicadas a la vida cotidiana. Grosso modo, esta cuarta generación puede subdividirse en tres grandes bloques de derechos, algunos de ellos derivaciones de otros ya anteriormente reconocidos y otros nacidos a partir de situaciones sin precedente. En un primer bloque, se encuentran los derechos relativos a la protección del ecosistema y el medioambiente, así como el patrimonio de la humanidad tangible e intangible. En el segundo bloque están los derechos relativos a un nuevo estatuto jurídico de la vida humana y en el tercero los derivados de las nuevas tecnologías de la comunicación y la información. Especial atención merecen los del segundo bloque, entre los que destacan y son relevantes al derecho a la identidad, los que defienden la integridad psicológica y moral del individuo, el derecho a la identidad genética y a recibir información sobre ella y el derecho a la reproducción humana (cfr. Yolanda Gómez Sánchez, Derecho Constitucional Europeo, Madrid, Sanz y Torres, 2005, pp. 60-68 passim).

33 James Griffin, On Human Rights, Oxford, Oxford University Press, 2009, pp. 152 y cc. 
legal. Esta vinculación se predica para todo operador del derecho, en especial el juez que debe asumir que el orden político y la paz social solo se fundan en el respeto a la dignidad de la persona. ${ }^{34}$

\subsection{Libre desarrollo de la personalidad: el cauce de la dignidad humana}

La función principal de la lucha por reconocer y consagrar jurídicamente una serie de derechos a favor del individuo y exigibles frente al Estado y los poderes públicos ha sido la de configurar y proteger una esfera de libertad individual en la que cada persona pueda decidir, con plena autonomía, cómo conformar sus opciones vitales. ${ }^{35}$ Los derechos fundamentales son concreciones del valor de la dignidad y esta, a su vez, goza de pleno respeto y eficacia cuando los derechos son honrados. ${ }^{36}$ Los derechos fundamentales, en definitiva, garantizan al sujeto su señorío sobre alguna parte esencial de su propia persona, y el ejercicio concreto de las proyecciones materiales y espirituales de diversas caras del todo integral que es la persona humana. ${ }^{37}$

Desde esta óptica, quizás uno de los más claros ejemplos normativos de cómo la dignidad humana actúa como fuente de derechos y exigencias propias de la naturaleza humana sea el artículo 19 de la Constitución Argentina. Existe una larga elaboración jurisprudencial con base en el reconocimiento y la protección de esferas de acción del individuo desde esta disposición constitucional, que abarca desde la interioridad del sujeto, ${ }^{38}$ hasta sus relaciones de familia. ${ }^{39}$ En línea con ello, vale mencionar el ejemplo de la Constitución Española de 1978 en su artículo 10.1 que menciona como fundamento del orden político y la paz social, la dignidad y el libre desarrollo de la personalidad.

En cuanto a la vinculación del concepto de dignidad con el de libre desarrollo de la personalidad, cabe señalar que la personalidad es el aspecto dinámico de la dignidad humana. ${ }^{40}$ El libre desarrollo de la personalidad se alcanza mediante la plena realización de las potencialidades humanas y el ejercicio de los derechos, especialmente los fundamentales. El reconocimiento y respeto de estos derechos se asienta, a su vez, en el reconocimiento y respeto de la dignidad. Es decir,

\footnotetext{
34 Mangas Martín (dir.), Carta de los Derechos Fundamentales de la UE, op. cit., pp. 99-101.

35 Yolanda Gómez Sánchez, Derecho Constitucional Europeo, Madrid, Sanz y Torres, 2005, pp. 58-59.

36 Rafael De Asís, “La apertura constitucional: la dignidad de la persona y el libre desarrollo de la personalidad como fundamentos del orden político y de la paz social”, en AA.VV., Comentarios a la Constitución Socioeconómica Española, Granada, Comares, 2002, pp. 154-156.

37 Joaquín Diez DíAz, en “¿Derechos de la personalidad o bienes de la persona?”, Revista General de Legislación y Jurisprudencia (1963), pp. 877 y 890.

38 Fallos 306:1892. Para un desarrollo de la interpretación dada en la doctrina y jurisprudencia al artículo 19 de la CN, véase la obra de Santiago Legarre, Poder de policía y moralidad pública, Buenos Aires, Ábaco de Rodolfo de Palma, 2004, pp. 244-278.

39 Fallos 308:2268.

40 María Corona Quesada González, “El derecho (¿Constitucional?) a conocer el propio origen biológico”, eN ANUARIO DE DERECHO CIVIL, T. I, ENERO-JUNIO 1994, P. 237.
} 
la dignidad es la cualidad de la cual derivan los derechos fundamentales y el ejercicio de estos implica el desarrollo de la personalidad. La dignidad contiene, en potencia, aquello que se despliega, en acto, con el proceso de perfeccionamiento y desenvolvimiento de la personalidad.

El libre desarrollo de la personalidad se integra también con el respeto a la autonomía individual. Tal vez detrás de este enunciado se encuentre la noción moral kantiana de tratar al hombre como fin y nunca como medio: el respeto a la dignidad humana implica el reconocimiento de la persona como ser independiente $\mathrm{y}$, con ello, el respeto de su personalidad e identidad. ${ }^{41} \mathrm{El}$ libre desarrollo de la personalidad se potencia y alcanza haciendo valer los derechos inviolables e inherentes a la persona. Estos derechos, más allá de la denominación que se les otorgue, son manifestaciones concretas del valor de la personalidad.

Dada la diversidad en la que puede manifestarse el derecho al libre desarrollo de la personalidad, y la multiplicidad de situaciones en las que la dignidad humana puede exigir su respeto y promoción, es que no puede crearse una lista cerrada que enumere taxativamente los derechos fundamentales. Asimismo, los derechos en concreto exigen esferas delimitadas y un alcance definido por cuanto implican zonas concretas de autonomía, lo que llama a analizar su contenido.

En virtud de estas razones es que el libre desarrollo de la personalidad, más que una cláusula de cierre de los derechos, actúa como una cláusula de reintegración que permite adaptar la lectura de las garantías constitucionales a la luz de los hechos y el devenir social con sus nuevas exigencias y desafíos. ${ }^{42}$ Siempre con la fuente y coto de la dignidad humana. Será la fuerza impulsora del cambio subjetivo y personal, en relación dialéctica con el cambio social y las concreciones históricas, las que irán delimitando la medida de la fórmula del "libre desarrollo de la personalidad". ${ }^{43}$

\section{La dignidad y la disposición sobre el propio cuerpo}

La dignidad del hombre se despliega y manifiesta en diversos niveles, todos ellos bajo el paraguas del libre desarrollo de la personalidad. ${ }^{44}$ De cada uno

41 Lara Aguado, El nombre en derecho internacional privado, op. cit., p. 79. Por identidad se entiende el derecho a ser uno mismo incluyendo todas las facetas en las que el ser se manifiesta, desde las individuales hasta las sociales, religiosas, culturales, lingüísticas, sentimentales y políticas, entre otras. Todo ello sin interferencias que desdibujen los trazos del yo.

42 Ibid., pp. 77-79.

43 Cfr. Rabbi-Baldi, Teoría del Derecho, op. cit., pp. 155-156, en la lúcida explicación que brinda de la elaboración de Llompart acerca de la constante interacción entre lo universal y la variabilidad histórica en la concreción de las exigencias de la dignidad humana.

44 Oscar Álzaga Villaamil, Comentarios a las leyes politicas: Constitución Española de 1978, t. II, Madrid, Revista de Derecho Privado, 1984, pp. 113-117. 
de estos cuatro niveles de despliegue derivan las libertades y los derechos reconocidos a las personas, tales como la libertad política y de expresión, la protección de la integridad física y psíquica, la intimidad y privacidad, la libertad religiosa, de creencias y de educación. ${ }^{45}$

Para empezar, la dignidad de la persona humana, en cuanto ser racional con capacidad de volición y autodeterminación, ha sido una constante en el pensamiento antropológico y filosófico de diversas culturas, con exponentes prístinos en la tradición judeo-cristiana. ${ }^{46}$ En segundo lugar, la dignidad se despliega en la pregunta por el origen y la finalidad de la propia existencia. Es la dimensión de lo religioso, con independencia de la creencia particular o respuesta que se dé a ese interrogante. En tercer lugar, otra manifestación de la dignidad humana se estima en la vida de relación y social de los individuos, en todo lo relacionado con su consideración y honor. En cuarto lugar, la dignidad es la dimensión moral o ética del hombre y de la mujer en cuanto a la valoración de los propios actos y sus consecuencias en relación con sí mismos y con terceros.

Esta cuarta acepción lleva al análisis de la disposición de una persona sobre su propio cuerpo, materia que habita y que constituye el sostén sobre el cual desarrolla su personalidad. ${ }^{47}$ En definitiva, toda persona alcanza su modelo de ser humano o ideal de vida en los confines de su cuerpo ${ }^{48}$ El valor intrínseco de la vida es asequible por la sola experiencia, se presenta al entendimiento como deseable per sey, en el plano del actuar ético, como razón suficiente para actuar. ${ }^{49}$

\footnotetext{
45 Ibid., p. 118.
}

46 Rabbi-BAldi, Teoría del Derecho, op. cit., pp. 61-62. Véase la cita de Picco della Mirandola con referencia a Zoroastres y Aristóteles. Algunos autores citan la influencia del pensamiento de Averroes en la elaboración del propio Picco della Mirandola y de Giordano Bruno en la composición del alma humana en perecedera y divina; así, la segunda es espejo de lo imperecedero y lo elevado.

47 Álzaga Villaamil, Comentarios a las leyes politicas: Constitución Española de 1978, op. cit., pp. 122-124.

48 Véase Javier Hervada, "Los trasplantes de órganos y el derecho a disponer del propio cuerpo", Persona $y$ Derecho 2 (1975), p. 220. De acuerdo con este autor, definimos a la vida en primer lugar como "un modo de ser, una mayor y mejor participación en el acto de ser [...] las distintas clases de vida [...] no son otra cosa que modos progresivos y superiores de poseer la perfección del acto de ser. En una segunda acepción y modo impropio, llamamos vida a las operaciones del ser vivo [...] y en este segundo sentido se habla de vida intelectual, amorosa, científica o cultural, política". Este modo de ser se corresponde con la naturaleza del viviente, $\mathrm{y}$, por ende, requiere una consideración no reduccionista, sino armonizante de todas las dimensiones del mismo. El hombre no es un ser puramente racional (res cogitans en la terminología de Descartes), ni tampoco materia pura (res extensa), sino una unidad de espíritu y cuerpo. Ya Platón marcó una visión dualista de la naturaleza humana, pero es en Descartes donde encontramos una visión del cuerpo humano como una máquina regida por las leyes de la física, al servicio del alma espiritual a la que se encuentra accidentalmente unido. Esta división desembocará con Marx y, en el existencialismo ateo de Sartre (J'existe mon corps, "yo existo mi cuerpo"), en un monismo materialista que agota al hombre en su corporeidad en tanto esta sea capaz de interacción con el medio. Deja de lado el despliegue de acciones humanas en un plano superior al sensitivo. El actuar humano no está condicionado por la respuesta automática al estímulo, sino que es final, proyectándose por sobre el hoy y el aquí. En el otro extremo del reduccionismo, encontramos al pensamiento liberal anglosajón, del que autores como Rawls y Dworkin son exponentes actuales, que considera a la vida como un mero instrumento para la realización del plan de vida (y de muerte) que cada individuo apto se imponga racionalmente. El individuo autónomo determina no solo la modalidad de vida, sino también su duración al ser esta un instrumento desechable. Olvida esta corriente que la vida es el sustrato sobre el cual se realiza el ser no mediatizable que es el hombre y que, por ende, posee valor intrínseco y no instrumental. Cfr. John M. Finnis, Natural Law and Natural Rights, Oxford, Clarendon Law Series, 1999, pp. 60-62. 
Y la importancia de la integridad física de ese cuerpo en el que se desarrolla la vida surge claramente como derivación necesaria del respeto a la vida misma.

Desde una perspectiva, la vida puede caracterizarse como el objeto de un derecho que cae dentro de los considerados básicos o fundamentales, y que revisten ciertas notas esenciales, entre ellas las de inviolabilidad e inalienabilidad. ${ }^{50}$ Estas cualidades son igualmente predicables del derecho a la integridad física por cuanto su respeto aparece inescindible de la preservación de la vida.

Mientras que el reconocimiento de esta categoría de derechos como inviolable tiende a asegurarlos frente al Estado y a los particulares, su conceptualización como inalienables busca subsanar la escisión entre derecho y deber provocada por el voluntarismo, y los salvaguarda frente al propio sujeto. ${ }^{51}$ En virtud de esta cualidad, el sujeto titular tiene vedados todos los actos de disposición física o jurídica, temporales o permanentes que suspendan o hagan imposible el ejercicio del derecho o bien signifiquen un atentado contra la dignidad, que es su fundamento y origen.

\subsection{La objeción de conciencia y el rechazo de tratamientos médicos}

Tomando las consideraciones anteriores, cabe analizar si la disposición que una persona hace sobre su propio cuerpo -en tanto manifestación concreta y tangible de su existencia- puede ser aceptada por el derecho en casos en que de esa decisión puedan derivarse consecuencias irremediables. Vale aclarar que el término irremediable se utiliza para significar algo de lo cual no existe vuelta al estado anterior de las cosas. ${ }^{.2}$

50 Como nos recuerda Spaemann, la dignidad es inviolable en el sentido de que no puede ser arrebatada desde afuera. La ausencia de respeto por esa cualidad no resulta en una pérdida de la misma para el sujeto afectado, sino en la gradual deshumanización del agresor.

51 Cfr. Antonio Luis Martínez-Pujalte, "Los derechos humanos como inalienables", en Jesús Ballesteros, Derechos Humanos, Madrid, Tecnos, 1992. Desde una segunda perspectiva de análisis, y a manera de refuerzo, es útil visualizar la teleología de este derecho desde la perspectiva histórica del pensamiento liberal y sus fundamentos filosóficos. Me permito así señalar la concordancia de aquellos pensadores en los que mayormente se funda la tradición liberal anglosajona. Hobbes retrotrae el fundamento de todos los derechos al más básico interés en la preservación de la propia vida. La búsqueda de la autoconservación lleva al hombre a "firmar" el pacto social, transfiriendo el ejercicio sobre la base de la fuerza de sus derechos al Leviatán -persona ficticia que ha de regular la convivencia social- a quien no puede desobedecer. Solo puede desobedecerse en justicia una orden de atentar contra la propia vida o de abstenerse de algo que sea indispensable para la subsistencia. Locke, al considerar la cuestión de un derecho natural al suicidio, lo descarta sobre la base de la inalienabilidad del dominio que todo hombre tiene sobre sí, a nadie le es permitido venderse como esclavo ni puede reconocérsele la libertad de suicidarse Ningún hombre puede disponer arbitrariamente de sí, en el más profundo de los sentidos, el derecho sobre la propia vida, integridad corporal y libertad se refiere a la facultad de excluir la disposición de terceros sobre los mismos, pero no confiere posibilidad alguna de autodisposición. La razón última de esta prohibición la encontramos, desde una perspectiva pragmática, en la necesidad de proteger al individuo de posibles abusos de los que pudiera ser víctima en situaciones límite. Piénsese en el caso de un sujeto que, llevado por la desesperación, decidiera venderse como esclavo. ¿Qué argumentos válidos existirían para detenerlo si gozase de una disponibilidad absoluta sobre su vida y libertad? El mismo Kant, en su Metafisica de las costumbres, se refiere a la ilicitud del suicidio: "el hombre no puede privarse de su personalidad en tanto esté unido por obligaciones, y en consecuencia mientras viva, y es una contradicción tener la facultad de librarse de toda vinculación".

52 Irremediable. Del lat. irremediab lis. 1. adj. Que no se puede remediar (Real Academia Española, Diccionario de la lengua española, Madrid, Espasa, 2014). 
Como primer tramo de análisis, se trae a consideración el supuesto en que una persona adulta y con plena capacidad manifiesta su negativa a recibir o proseguir un tratamiento médico. Ello en tanto expresa que el inicio o la prosecución de ese tratamiento le significaría un dilema ético o, directamente, incurrir en un acto contrario a su creencia religiosa. Se trata de una situación que el máximo tribunal argentino ha tenido ocasión de considerar en dos oportunidades, separadas entre sí por dos décadas. ${ }^{53}$ En concreto, los precedentes "Bahamóndez" y "Albarracini Nieves". 54

Una relación sucinta del precedente "Bahamóndez" indica que este ingresa a un hospital público, siendo mayor de edad y en pleno uso de sus facultades, con una severa hemorragia cuyo tratamiento debe ser una inmediata transfusión de sangre. El paciente se rehúsa, con total conocimiento de las posibles consecuencias fatales de su negativa, y aduce profesar el culto de los Testigos de Jehová. En sus propias palabras, asegura que no desea morir, pero tampoco vivir de manera contraria a sus íntimas convicciones. La situación es judicializada a partir del pedido de los profesionales médicos intervinientes.

El caso anterior difiere de "Albarracini Nieves" en que, en este segundo precedente, del año 2012, el paciente es una persona mayor de edad que ingresa en estado inconsciente a una guardia tras sufrir una agresión con arma de fuego que lo deja al borde de la muerte. Los galenos prescriben transfusiones de sangre y la cónyuge del Sr. Albarracini produce un documento en que, bajo el instituto de las directivas anticipadas de la Ley 26.529, el paciente ha dejado asentada su negativa a toda terapéutica contraria a los preceptos del culto de los Testigos de Jehová.

En el primer precedente, la Corte Suprema de Justicia de la Nación (CSJN) afirmó que

... la posibilidad de que los individuos adultos puedan aceptar o rechazar libremente toda interferencia en el ámbito de su intimidad corporal es un requisito indispensable para la existencia [...] de la autonomía individual [excepto en el caso de] que exista algún interés público relevante en juego y que la restricción al derecho individual sea la única forma de tutelar dicho interés.

Siguiendo este razonamiento, veinte años después, la Corte afirma que "aceptar o rechazar un tratamiento específico, o de seleccionar una forma alternativa de tratamiento hace a la autonomía y autodeterminación; que los pacientes tienen derecho a hacer de acuerdo con sus propios valores o puntos de vista". Reitera los mismos límites explicados en el año 1993. En ambos supuestos, el tribunal es claro en afirmar que no se trata de un permiso para el suicidio, sino del ejercicio de las convicciones personales en una esfera que, incluso en

53 Véanse los distintos análisis efectuados en Renato Rabbi-Baldi Cabanillas (coord.), Los derechos individuales ante el interés general, Buenos Aires, Ábaco de Rodolfo Depalma, 1998, pp. 157-187. 
un espacio público como es un centro sanitario, sigue permeada del carácter de privado del artículo 19 de la Constitución Nacional.

Adentrándonos en los límites, las diferencias entre tratamientos médicos (respiradores artificiales, nutrición artificial, diálisis o un simple tratamiento para la gripe), ya sea en lo que hace a su aplicabilidad como a sus resultados, son evidentes. No puede sostenerse, razonablemente, que todas las terapéuticas son equiparables. Calificar una conducta depende de la: i) intencionalidad del agente; ii) del nexo de causalidad que la une con el resultado y, en el caso de los tratamientos médicos; iii) si importa o no la abstención de medios proporcionados o desproporcionados al respeto por la dignidad del paciente..$^{55}$ Es necesario, entonces, analizar las diferencias tanto objetivas como subjetivas que existen en cada caso para así poder distinguir entre un acto dirigido a terminar la vida y el rechazo de una terapéutica que resulta una afrenta a alguna de las dimensiones del libre desarrollo de la personalidad del paciente.

En el plano de la intención, cabe considerar la conciencia del paciente; así, el que se niega a un tratamiento argumentando convicciones religiosas tiene por objeto alinear su vida interior e integridad espiritual con su existencia corporal. No busca la consecución del resultado muerte, y la disminución física que pueda eventualmente derivarse de su decisión no es el fin de su acción, sino una consecuencia tolerada. La mira de su actuar está centrada en el pleno despliegue de todas las facetas de su personalidad en la mayor extensión posible. ${ }^{56}$

Desde la dimensión objetiva del actuar, la negativa a proseguir una terapéutica en un caso terminal -o potencialmente irreversible- exige, en sentido material, la existencia de un proceso subyacente que esté siendo frenado por el tratamiento en cuestión. La causa eficiente del daño no está dada por la ausencia del tratamiento, sino por un factor anterior al que el sujeto ha sido ajeno. Al retirarse o no oponerse las barreras médicas, el curso que ya había comenzado prosigue su natural desenvolvimiento.

Este segundo factor o consideración objetiva de lo que implica la disposición del propio cuerpo mediante la negativa a recibir o proseguir una terapéuti-

55 Según como se conjuguen los tres aspectos, nos encontraremos en un supuesto de eutanasia, ortotanasia o encarnizamiento terapéutico. Por eutanasia se entiende una acción positiva u omisiva que introduce el factor muerte, ya sea por voluntad del que padece (eutanasia voluntaria), o del que la inflige (eutanasia involuntaria). La ortotanasia, por el contrario, envuelve la no aplicación o suspensión de terapéuticas incapaces de detener la vida u obtener resultados proporcionados a las exigencias del tratamiento. La prolongación artificial de la vida, que convierte al cuerpo humano en una máquina cuando no hay perspectiva alguna de mejoría, es lo que se conoce como encarnizamiento terapéutico (cfr. Elio SGRECCIA, Manual de bioética, Diana, México D.F., 1992). Es de aplicación la doctrina del "acto de doble efecto", cuando una acción orientada a un fin bueno -en este caso el cese de sufrimiento físico o padecimientos morales- se encuentra inescindiblemente ligada a consecuencias negativas previsibles. Será lícito llevar a cabo la acción, sea esta positiva o negativa, en tanto se cumplan las siguientes condiciones: i) que la acción buscada sea buena; ii) que el efecto bueno sea el directamente buscado; iii) que el efecto malo no sea querido como fin; y iv) que se observe una proporción entre la bondad directamente querida y el mal tolerado. 
ca encuentra su fundamento en la noción de dignidad inherente y fundante analizada en los apartados anteriores. Ello conduce a un análisis de proporcionalidad en relación con la dignidad del sujeto sobre el cual se propone la terapéutica en cuestión. Un claro ejemplo de esto lo brindan los incisos g) y h) del actual artículo 59 del Código Civil y Comercial argentino. ${ }^{57}$ Es necesario tener en cuenta la integridad del ser humano, cuya vida no implica ni una mera persistencia biológica ni únicamente puede ser reducida a algunas funciones corporales. No debe perderse de vista que el paciente es un todo, el cual exige que una terapia se valore teniendo en cuenta una serie de factores. Y, más aún, la consideración de la dignidad y derechos de los terceros.

En otras palabras, la disposición que se hace del cuerpo por parte del sujeto no significa un menoscabo a su dignidad en tanto la acción no consiste en una mediatización de sí mismo o de terceros. La vivencia de las elecciones interiores-las más libres de todas- por parte de la persona conlleva -como efecto no deseado, pero factible- un riesgo para la salud o la vida. El sujeto no origina ese riesgo, no busca menoscabar su integridad física, sino dar plena exaltación a su vida interior que es aquello más característico de lo humano entendido como Digno. Si tomamos la definición previamente estipulada, ${ }^{58}$ vemos que en el obrar de Bahamóndez y Albarracini Nieves hay una autodeterminación consciente de la propia vida.

\subsection{Maternidad subrogada y extensión de la libre disponibilidad sobre el cuerpo propio}

La maternidad subrogada es una de las figuras más discutidas en el campo de la reproducción humana con auxilio de técnicas médicas. Si bien es usual la referencia a la práctica de la maternidad subrogada como una técnica de reproducción humana asistida (TRHA), su estructura hace que se acerque más a un supuesto de voluntariedad en la supresión y atribución de la filiación materna que a un caso típico de reproducción asistida. Aunque la maternidad subrogada como práctica tiene en común con las TRHA de tipo heterólogo el

57 "Artículo 59. Consentimiento informado para actos médicos e investigaciones en salud. El consentimiento informado para actos médicos e investigaciones en salud es la declaración de voluntad expresada por el paciente, emitida luego de recibir información clara, precisa y adecuada, respecto a [...] g) en caso de padecer una enfermedad irreversible, incurable, o cuando se encuentre en estado terminal, o haya sufrido lesiones que lo coloquen en igual situación, el derecho a rechazar procedimientos quirúrgicos, de hidratación, alimentación, de reanimación artificial o al retiro de medidas de soporte vital, cuando sean extraordinarios o desproporcionados en relación a las perspectivas de mejoría, o produzcan sufrimiento desmesurado, o tengan por único efecto la prolongación en el tiempo de ese estadio terminal irreversible e incurable; h) el derecho a recibir cuidados paliativos integrales en el proceso de atención de su enfermedad o padecimiento".

58 La dignidad como "un valor espiritual y moral inherente a todas las personas, un mínimo que debe preservarse de las vulneraciones por parte de los poderes públicos y los particulares. Se manifiesta en la autodeterminación consciente y responsable de la propia vida, en la autonomía individual y constituye el punto de partida para la existencia y especificidad de los demás derechos fundamentales". (Humberto Nogueira Alcalá, "El derecho a la propia imagen como derecho fundamental implícito. Fundamentación y caracterización", en Ius et Praxis 13-2 [2007], pp. 245-285). 
involucrar la cooperación de un tercero -la madre sustituta o gestante- presenta dos notas que la diferencian esencialmente de la donación de gametos o embriones. En la maternidad subrogada están ausentes la nota de fungibilidad del aporte del tercero a la concepción de la criatura, y la colaboración de quien aporta el elemento heterólogo al proceso de gestación y alumbramiento es continuada y no reducida a un solo acto de disposición del material genético. ${ }^{59}$

Esto se traduce en que no hay posibilidad de fragmentación física, psicológica y afectiva de la participación de la mujer gestante. A diferencia del donante de gametos que separa de su cuerpo un producto, la gestante pone la integridad de su cuerpo de mujer para la anidación, la gestación y el parto. ${ }^{60}$

Al sancionarse la Ley 26.994, el legislador argentino optó por excluir del nuevo articulado civil la figura de la maternidad subrogada y su encuadre normativo. Con anterioridad a la entrada en vigencia del Código Civil y Comercial de la Nación, el Primer Juzgado de Familia, Primera Circunscripción Judicial de la provincia de Mendoza atribuyó la maternidad sobre una criatura a la mujer que aportó sus óvulos para que el niño fuera gestado por una tercera. ${ }^{61} \mathrm{La}$ decisión judicial fue favorable a la pretensión de los actores en tanto declaró inaplicable al caso en cuestión el entonces vigente artículo 242 del Código Civil, y que la filiación debía determinarse de acuerdo con su origen genético y, fundamentalmente, la voluntad procreacional manifestada por los comitentes. El fundamento de la decisión estribó, principalmente, en el elemento de la voluntad procreacional manifestada que se sumó a la disposición de lo que consideró era "el propio útero" con la finalidad de llevar adelante una gestación.

Con posterioridad a la entrada en vigencia del Código Civil y Comercial el 30 de diciembre de 2015, el Juzgado de Familia 7 de Lomas de Zamora, provincia de Buenos Aires, declaró la inconstitucionalidad y anticonvencionalidad del artículo 562 del Código Civil y Comercial argentino. ${ }^{62}$ Ello en virtud de no

59 Esto es, las TRHA en las que hay intervención de terceros se caracterizan por la fungibilidad del aporte de estos terceros y porque esta cooperación se agota en un único acto. Así, el semen y los óvulos donados son fungibles en tanto quienes habrán de recibirlos pueden llevar a cabo su tratamiento con una muestra u otra. No es así en el caso de un embarazo por encargo en el que la pareja comitente escoge a una mujer en particular para gestar un embrión. La identidad y particularidades de la mujer portadora son conocidas por el equipo médico y por los padres intencionales y es a ella y no a otra a quien desean recurrir. Por ello mismo, la colaboración de esta mujer no se agota en un único acto de entrega de gametos, sino que posee una activa participación en la planificación de la concepción, el embarazo y el parto. Por otro lado, en la maternidad de sustitución la mujer que gesta al hijo adquiere un vínculo biológico y afectivo con él durante los nueve meses de embarazo, a diferencia de los donantes de gametos masculinos o femeninos cuya colaboración se reduce a un acto concreto de aporte de sus gametos sin conocer el desarrollo ulterior del proceso de fecundación y gestación, y es conocida por los comitentes.

60 Mercedes Ales Uría, “La maternidad por acuerdo de partes”, El Derecho, 29 de diciembre de 2012, Año XLIX, N ${ }^{\circ} 12.906$ y "Legislar sobre la maternidad subrogada”, en coautoría con Úrsula C. BAssET, Revista La Ley, (2018). También "Maternidad subrogada: vulnerabilidad legal y social. Situación actual en Argentina. La mirada en el espacio europeo", en Úrsula C. BAsset, Hugues Fulchiron et al. (dirs.), Tratado de la vulnerabilidad, Buenos Aires, La Ley, 2017.

61 Expte. 714/15, “O.A.V., G.A.C y F.J.J. por medida autosatisfactiva”, sentencia de 29 de julio de 2015.

62 Expte. LZ-62420-2015, “H.M. y Otro S/medidas precautorias (art. 232 CPCC) (419)”, Juzgado de Familia 7, Lomas de Zamora, sentencia de 30 de diciembre de 2015. 
reconocer la maternidad de la mujer que hubiere expresado su voluntad procreacional mediante consentimiento informado y determinar la maternidad por el hecho del parto en todos los casos. ${ }^{63}$

Entre la jurisprudencia que cita el Juzgado de Familia que toma conocimiento del caso cita se encuentran los precedentes Menesson c. Francia y Labasse c. Francia resueltos por el Tribunal Europeo de Derechos Humanos (TEDH) en el año 2014..$^{64}$ Los extremos fácticos en los dos casos guardaban estrecha similitud ya que se trataba del reclamo de reconocimiento del vínculo filial -y con ello la ciudadanía y los derechos conexos- de menores de edad nacidas en Estados Unidos mediante acuerdos de gestación. En el país de nacimiento la filiación había sido determinada a favor de los comitentes, de forma contraria a las disposiciones de orden público francés. Por este motivo, la República de Francia se oponía a la inscripción registral de los menores como hijos de los comitentes. En el litigio contra el Estado francés, ambos grupos de demandantes alegaron violación del artículo 8 del Convenio Europeo de Derechos Humanos (CEDH) en cuanto protege la vida familiar.

Lo cierto es que en Menesson y Labasee el TEDH encontró que el Estado francés había violado la protección de la vida familiar de los menores, no así de los adultos. Es decir, la visión del TEDH se centró en la situación que en los hechos se había provocado con respecto de las menores. En ningún momento se afirmó que el no reconocimiento de la figura de la gestación por sustitución fuese violatorio del derecho a la vida familiar de los adultos que desean recurrir a ella. Es más, en los considerandos del fallo Menesson se releva un dato objetivo y elocuente en materia de la falta de consenso sobre esta figura: de 35 estados relevados únicamente siete permiten de manera expresa la maternidad subrogada. ${ }^{65}$ Ambos precedentes afirmaron la libertad de cada estado parte del CEDH para legislar en materia de gestación por subrogación.

Es importante destacar que, tanto en las sentencias locales como en las del TEDH, se presentaron ante los tribunales supuestos post facto o hechos consumados: niños ya concebidos y nacidos -o a pocas semanas de nacer- en situación potencial o actual de total vulnerabilidad jurídica (ausencia de madre dispuesta a asumir la responsabilidad parental, problemas de nacionalidad y apatridia, etc.). En los cuatro precedentes está patente la necesidad de proteger al niño. La jurisprudencia europea es clara en cuanto a que la salvaguardia del niño no legitima el origen de su nacimiento.

63 Posteriormente, los tribunales argentinos han prodigado una decena más de casos sobre el tema. Para un análisis de estos y una crítica al activismo judicial en la materia, véase Mercedes AlEs Uría, "Argentina's absence of regulation of surrogate motherhood: A case of judicial activism and unforeseeable consequences", en AA.VV., Family Law and Family Realities, Netherlands, Seven Publishing, 2019.

5864 Ambas sentencias del TEDH de fecha 26 de junio de 2014, identificadas con los números 65192/11 y 65192/11 respectivamente.

65 Para una lectura en clave diferente de estos precedentes, véase Marisa Herrera y Eleonora Lamm, “Un valiente fallo del TEDH sobre gestación por sustitución. Prohibir, silenciar, regular o fallar", La Ley (2014-D), p. 1165. 
Asimismo, en el espacio europeo, en su Resolución de 17 de diciembre de 2015, el Parlamento ha afirmado que la práctica de gestación por sustitución

\begin{abstract}
... es contraria a la dignidad humana de la mujer, ya que su cuerpo y sus funciones reproductivas se utilizan como una materia prima [por lo que] debe prohibirse esta práctica, que implica la explotación de las funciones reproductivas y la utilización del cuerpo con fines financieros o de otro tipo, en particular en el caso de mujeres vulnerables en los países en desarrollo. ${ }^{66}$
\end{abstract}

Esta decisión es concordante con la Resolución del Parlamento Europeo del 5 de abril de 2011 sobre prioridades y esquema del nuevo marco de políticas de la Unión Europea para luchar contra la violencia contra la mujer. En ella se llamó a los Estados miembros a reflexionar sobre "el serio problema de la subrogación que constituye una explotación del cuerpo femenino y sus órganos reproductivos", y enfatizaba que "las mujeres y los niños están sujetos a las mismas formas de explotación y ambos pueden ser vistos como commodities" ${ }^{67}$

En el marco de análisis propuesto al examinar el acto de negativa a recibir tratamiento médico, incluso cuando de ello dependiera la propia subsistencia, cabe preguntarse si el empleo que una mujer hace de su propia persona bajo un acuerdo de maternidad subrogada se encuentra abarcado dentro de la disposición del propio cuerpo que se sustenta en la dignidad humana. Ello desde la triple perspectiva propuesta: objetiva, subjetiva y de proporcionalidad.

Desde la dimensión objetiva del actuar, la gestación por subrogación exige que la totalidad del ser humano sea puesta a disposición del triple objetivo de concebir, gestar y parir. La medicina y la experiencia humana ponen de manifiesto que el proceso de embarazo no involucra un órgano aislado del cuerpo de la mujer gestante -su útero-, sino que las dimensiones físicas y psíquicas se vuelcan en su totalidad a la gestación del embrión, posterior feto y luego niño. Estudios recientes han demostrado, incluso, que el ADN del feto puede ser encontrado en el cerebro materno décadas después del nacimiento. ${ }^{68}$

Esa disposición total no tiene por resultado la cura de la esterilidad o patología padecida por la mujer que es sustituida como gestante, sino la generación de un niño vivo. En otras palabras, concluido el acuerdo, la mujer imposibilita-

66 Véase párrafo 115, en http://www.europarl.europa.eu/sides/getDoc.do?pubRef=-//EP//TEXT+TA+P8-TA2015-0470+0+DOC+XML+V0//ES

67 Véanse párrafos 20 y 21, en http://www.europarl.europa.eu/sides/getDoc.do?pubRef=-//EP//TEXT+TA+P7TA-2011-0127+0+DOC+XML+V0//ES

68 William Chan et al., "Male microchimerism in the human female brain", Ed. Martin Gerbert Frasch. PLoS ONE 7.9 (2012): e45592. PMC, en https://www.ncbi.nlm.nih.gov/pmc/articles/PMC3458919/. Excede la extensión de este trabajo, si bien guarda estrecha vinculación con su temática, la discusión en torno al ADN fetal y la configuración de la persona en términos jurídicos que se ha dado en los casos de pretensiones de interrupción del embarazo por tratarse de gestaciones de fetos anencefálicos. Véase el fallo de la CSJN, Tanus, S., c/ Gobierno de la Ciudad de Buenos Aires, de 2 de enero de 2001. 
da para gestar no habrá superado su patología. La intervención médica no ha resuelto una carencia, sino que habrá entregado un resultado.

En el plano de la intención, cabe considerar la motivación de la mujer que presta su persona para la gestación de un niño que será entregado a un tercero. La práctica internacional de los países en los que la gestación por subrogación se encuentra regulada, ejemplifica que las transacciones que se llevan a cabo suelen tener una motivación económica por parte de la gestante. Es decir que la gestación es considerada como una locación de un servicio que implica a la casi totalidad de la propia persona con una finalidad eminentemente lucrativa. La mujer no acepta un embarazo con la finalidad de desarrollar su identidad en el plano de la maternidad: busca gestar un niño para entregarlo al cuidado y responsabilidad parental de otro.

Finalmente, desde la óptica de la dignidad, vale reiterar que esta implica las dimensiones biológica y afectiva del sujeto gestante. La mujer que presta su cuerpo a la gestación de un niño para otro, es un todo personal. No pareciera forzarse la fórmula kantiana de respeto por la persona al afirmar que la locación de ese todo que es la persona de la gestante significa la mediatización del sujeto. Por ello, todo lo opuesto a la afirmación del valor de la dignidad como fundamento de los derechos, incluyendo el de disposición del propio cuerpo. Y, más aún, la consideración de la dignidad y los derechos de los terceros, en particular del niño que resulta de la gestación. ${ }^{69}$

La negación de la dignidad ocurre en los dos sujetos principalmente involucrados: la gestante y el niño o la niña por nacer o por ser concebido. En la mujer, esta es vista como un medio para la obtención de un fin: la satisfacción de cierta comprensión del derecho a la reproducción del o los comitentes. La prestación del propio cuerpo para la reproducción de otro, usualmente además a partir de la recepción de un precio, significa la reducción del ser humano gestante a una maquinaria. Por otra parte, en lo que concierne a la persona por ser concebida y gestada, esta se reduce al resultado de un proceso. Es conceptualizada como el objeto de un derecho subjetivo: el derecho a la reproducción de los padres intencionales. ${ }^{70}$

No existe un "derecho al hijo" bajo los estándares de derecho internacional; ${ }^{71}$ ello en tanto un hijo no es un bien, servicio o potestad que pueda ser objeto de

$69 \quad$ Acerca de la situación legal de la maternidad subrogada en el espacio europeo y los conflictos de leyes que esta figura presenta puede consultarse el informe del Parlamento Europeo, Dirección General de Políticas Interiores, "El régimen de subrogación en los Estados miembros de la UE", 2013, disponible en http://www. europarl.europa.eu/RegData/etudes/etudes/join/2013/474403/IPOL-JURI_ET(2013)474403(SUM01)_ES.pdf

70 Mercedes Ales Uría, “¿Voluntad procreacional o derecho al hijo”, Revista de Derecho de Familia y de la Persona 4 (2019), p. 244.

71 El reporte cita a Chantal SAClier, “Children and adoption: Which rights are whose?”, Innocenti Digest 4, Florence, Unicef, 1999, pp. 12-13; Geraldine van Bueren, The International Law on the Rights of the Child, Amsterdam, Kluwer Law International, 1998. 
un derecho tutelado por el Estado. El hijo es un sujeto de derecho. Por ende "proveer de un derecho al hijo sería una negación fundamental de los iguales derechos humanos del hijo. La aproximación del 'derecho al hijo' debe ser resistida vigorosamente, ya que socaba la premisa fundamental de que los niños son personas con derechos humanos". ${ }^{72}$

Existen, a su vez, diversas objeciones desde el plano principialista ius filosófico -en las que confluyen diferentes vertientes de pensamiento- por las cuales los acuerdos de maternidad subrogada no deberían ser aceptables dentro del plano de la autonomía personal y alcance del derecho a la reproducción. ${ }^{73}$ Ello por cuanto se trata de actos de la voluntad que sujetan a determinadas personas -la mujer gestante y el potencial hijo- a la calidad de objeto de derecho. Esto adquiere mayor relevancia cuanto mayor es la sujeción de unos a la potestad de otros: en casos como el que se analiza, se confiere a un sujeto de adulto el "derecho" a decidir la existencia de otro ser humano y controlar de forma absoluta su inicio, desarrollo y hasta su ADN.

\section{Conclusiones}

El concepto de dignidad es de difícil determinación y continúa siendo fuente de ricos debates en el campo de la filosofía, la ética y el derecho. En este trabajo se abordó y definió desde una filiación kantiana en el sentido de la capacidad inherente a todo ser humano de autodeterminarse de manera consciente y conforme la propia comprensión ética. La dignidad como título o fundamento de los derechos encuentra un cauce adecuado en la elaboración del "libre desarrollo de la personalidad". Los derechos tienen causa en la dignidad, pero también encuentran en ella sus límites. Es decir, aquello que es fuente es también patrón de medida: no puede afirmarse la extensión de un derecho en actos que representen la negación de los elementos propios de la dignidad humana.

La elaboración que hacen la bioética y la jurisprudencia constitucional del derecho al desarrollo de la personalidad a través de las acciones que implican

72 El reciente Reporte de la Informante Especial de la Organización de las Naciones Unidas (ONU) sobre trata y explotación sexual, incluyendo prostitución y pornografía infantil, de fecha 15 de enero de 2018 ("Report of the Special Rapporteur on the sale and sexual exploitation of children, including child prostitution, child pornography and other child sexual abuse material" (A/HRC/37/60), presentado el 15 de enero de 2018 ante la Asamblea General de las Naciones Unidas, en https://documents-ddsny.un.org/doc/UNDOC/ GEN/G18/007/74/PDF/G1800774.pdf?OpenElement, abordó el tema de la maternidad subrogada tanto en su faceta comercial como altruista. El abordaje de este documento parte de la prístina concepción de que los instrumentos de derechos humanos, tanto internacionales como regionales y supranacionales, protegen el derecho a "fundar una familia", el derecho al "respeto por la vida privada y familiar" (en el cuerpo de este trabajo, párr. 64 del Reporte) (Traducción propia). Véase el detallado análisis de dicho documento efectuado en Úrsula C. BASSET, "La maternidad subrogada como trata y explotación de niños. Informe oficial de la Asamblea General de la ONU", La Ley, 11 de septiembre de 2018.

73 Véase el análisis que efectúa en este aspecto Hugo Oscar Seleme, "La maternidad por subrogación y los límites de la autonomía", La Ley, 18 de enero de 2013. También Spaemann, "Sobre el concepto de dignidad humana", op. cit., pp. 29-32. 
una disposición -total o parcial- del propio cuerpo sirve para ilustrar el sutil equilibrio entre la fundamentación y la limitación que es la dignidad humana. Tal es el caso del derecho a disponer del propio cuerpo mediante la negativa a someterse a ciertas terapéuticas que ofrece la ciencia médica con fundamento en las creencias del sujeto sobre cuya corporeidad deberían efectuarse. La extensión de este derecho se valora desde la subjetividad de la persona en cuanto concreta creencias y vivencias internas del sujeto; y también desde la objetividad en la medida en que los actos a los que aspira la persona no son causa eficiente de daños o perjuicios a sí mismo, a intereses públicos o a terceros. Reafirmar la dignidad inherente al titular del derecho estriba en considerar al ser humano como esa unidad indisoluble de biología y espíritu.

Por el contrario, en el caso de la disposición que una mujer hace de sí misma en el acuerdo de maternidad subrogada, no existe una afirmación de la dignidad humana, sino una objetivización de su persona y de la persona que habrá de gestar: se coloca en la posición de una maquinaria al servicio de un proceso que deberá entregar un producto que cumpla con las especificaciones deseadas por los comitentes. Implica la utilización del cuerpo y la psique para obtener un resultado que queda fuera de la vida de la mujer al entregar al ser humano, fruto de su vientre, a terceros en el contexto de una transacción. Se trata de actos negatorios de la dignidad en tanto cualidad del ser humano de ser fin en sí mismo.

La razón última de las notas de inviolabilidad e indisponibilidad de los derechos humanos se encuentra en su mismo fundamento: la dignidad de su titular, que Kant traduce en la exigencia de no mediatización del hombre, extensible tanto a los otros como al mismo sujeto, y que estriba en su carácter personal, es decir, racional y libre.

\section{Bibliografía}

Ales Uría, Mercedes, "La maternidad por acuerdo de partes", El Derecho XLIX (12.906) (2012).

Ales Uría, Mercedes, "Voluntad procreacional o derecho al hijo", en Revista de Derecho de Familia y de la Persona 4 (2019), p. 244.

Ales URía, Mercedes, "Legislar sobre la maternidad subrogada”, en coautoría con BASSET, U.C., en La Ley (2018).

Ales Uría, Mercedes, "Maternidad subrogada: vulnerabilidad legal y social. Situación actual en Argentina. La mirada en el espacio europeo", en U. C. Basset, H. Fulchiron et al. (dirs.), Tratado de la vulnerabilidad, Buenos Aires, La Ley, 2017. 
Álzaga Villaamil, Oscar, Comentarios a las leyes políticas: Constitución Española de 1978, t. II, Madrid, Revista de Derecho Privado, 1984, pp. 113-117.

BARILÁN, Yechiel Michael, Human dignity, human rights and responsibility, Cambridge, Massachussets Institute of Technology Press, 2012.

Castán Tobeñas, José, Los derechos del hombre, 2ª ed., Madrid, Reus, 1976.

Chan, William et al., "Male microchimerism in the human female brain", en Martin Gerbert Frasch, PLoS ONE 7.9 (2012): e45592. https://doi. org/10.1371/journal.pone.0045592

De Asís, Rafael, "La apertura constitucional: la dignidad de la persona y el libre desarrollo de la personalidad como fundamentos del orden político y de la paz social", en AA.VV., Comentarios a la Constitución Socioeconómica Española, Granada, Comares, 2002, pp. 154-156.

De Castro y Bravo, Francisco, "Los llamados derechos de la personalidad", en Anuario de Derecho Civil, 1959, pp. 1237, 1252, en https://www.boe.es/publicaciones/anuarios_derecho/articulo.php?id=ANU-C-1959-40123701276

De Miguel Beriain, Íñigo, “Consideraciones sobre el concepto de dignidad humana", en Anuario de filosofia del derecho 21 (2004), pp. 187-212, en https://dialnet.unirioja.es/servlet/articulo?codigo $=1217052$

DicкE, Klaus, "The founding function of human dignity in the Universal Declaration of Human Rights", en David KRETzMER y Eckart KLEIN (eds.), The Concept of Dignity in Human Rights Discourse, The Hague, Kluwer Law International, 2002, pp. 111-118.

Diez Díaz, Joaquín, “¿Derechos de la personalidad o bienes de la persona?”, en Revista General de Legislación y Jurisprudencia, (1963), pp. 877 y 890.

FinNis, John M., Natural Law and Natural Rights, Oxford, Clarendon Law Series, 1999.

Garrido Falla, Francisco, Comentarios a la constitución, Madrid, Civitas, 1985.

Gómez SÁnchez, Yolanda, Derecho constitucional europeo, Madrid, Sanz y Torres, 2005.

Griffin, James, On Human Rights, Oxford, Oxford University Press, 2009.

Herrera, Marisa y Eleonora Lamm, "Un valiente fallo del TEDH sobre gestación por sustitución. Prohibir, silenciar, regular o fallar", Buenos Aires, La Ley (2014-D), p. 1165.

Hervada, Javier, "Los trasplantes de órganos y el derecho a disponer del propio cuerpo", en Persona y Derecho 2 (1975), p. 220, en https://www.unav.edu/ publicaciones/revistas/index.php/persona-y-derecho/article/view/32805 
Kant, Immanuel, Fundamentación de la metafisica de las costumbres, Madrid, Espasa Calpe, trad. de M. García Morente,1983.

LARA Aguado, Ángeles, El nombre en derecho internacional privado, Granada, Comares, 1998.

Legarre, Santiago, Poder de policía y moralidad pública, Buenos Aires, Ábaco de Rodolfo de Palma, 2004.

Lucas Verdú, Pablo, “Comentario al art. 10”, en AA.VV., Constitución Española, Madrid, Centro de Estudios Constitucionales, 1979.

Mangas Martín, Araceli (dir.), Carta de los derechos fundamentales de la UE, Bilbao, Fundación BBVA, 2008.

Martín Bernal, José Martín, "Los derechos de la personalidad en la Constitución Española", en Revista General de Legislación y Jurisprudencia (1979), p. 13.

Martinez-Pujalte, Antonio Luis, "Los derechos humanos como inalienables", en J. Ballesteros (ed.), Derechos humanos, Madrid, Tecnos, 1992.

Micheal, Lucy, "Defining dignity and its place in human rights", en The New Bioethics 20 (1) (2014), pp. 12-34, en https://www.ncbi.nlm.nih.gov/pubmed/24979874

Nogueira Alcalá, Humberto "El derecho a la propia imagen como derecho fundamental implícito. Fundamentación y caracterización", en Ius et Praxis, vol. 13, núm. 2, 2007, pp. 245-285.

Peces Barba, Gregorio, "La dignidad de la persona desde la filosofía del derecho", en Cuadernos Bartolomé de las Casas 26 (2002), pp. 21-61, en https://www.dykinson.com/libros/la-dignidad-de-la-persona-desde-lafilosofia-del-derecho/9788497722346/

Quesada González, María Corona, “El derecho (¿constitucional?) a conocer el propio origen biológico", en Anuario de derecho civil, t. I, enero-junio 1994.

RabBi-Baldi Cabanillas, Renato (coord.), Los derechos individuales ante el interés general, Buenos Aires, Ábaco de Rodolfo Depalma, 1998, pp. 157-187.

Rabbi-Baldi Cabanillas, Renato, Teoría del Derecho, 4ae ed., Buenos Aires, Ábaco de Rodolfo de Palma, 2015.

Rogel Vide, Carlos, Bienes de la personalidad, derechos fundamentales y libertades públicas, Bolonia, Publicaciones del Real Colegio de España, 1985.

Salvador Gutiérrez, Susana, "Derecho a la identidad", en Actualidad Civil IV (1999), pp. 1469-1498. 
SEleme, Hugo Oscar, "La maternidad por subrogación y los límites de la autonomía", en La Ley, 2013.

SchACHTER, Oscar, "Human dignity as a normative concept", en American Journal of International Law 77 (1983), p. 848. https://doi.org/10.2307/2202536

Sgreccia, Elio, Manual de Bioética, México D.F., Diana, 1992.

Spaemann, Robert, "Sobre el concepto de dignidad humana", en Persona y Derecho19 (1988), pp. 13-33, en https://www.unav.edu/publicaciones/revistas/index.php/persona-y-derecho/article/view/32580

Waldron, Jeremy, "Is dignity the foundation of human rights", en New York University Public Law and Legal Theory Working Papers, Paper 374 (2013), en https://www.peacepalacelibrary.nl/ebooks/files/36335655X.pdf 